\title{
Blue light-dependent changes in loosely bound calcium in Arabidopsis mesophyll cells: an X-ray microanalysis study
}

\author{
Justyna Łabuz ${ }^{1,2}$, Sławomir Samardakiewicz ${ }^{3}$, Paweł Hermanowicz ${ }^{1}$, Elżbieta Wyroba ${ }^{4}$, Maria Pilarska ${ }^{1, \dagger}$ and \\ Halina Gabryś ${ }^{1, *}$ \\ ${ }^{1}$ Department of Plant Biotechnology, Faculty of Biochemistry, Biophysics and Biotechnology, Jagiellonian University, Krakow, Poland \\ ${ }^{2}$ Malopolska Centre of Biotechnology, Jagiellonian University, Krakow, Poland. \\ ${ }^{3}$ Laboratory of Electron and Confocal Microscopy, Faculty of Biology, Adam Mickiewicz University, Poznań, Poland. \\ ${ }^{4}$ Laboratory of Electron Microscopy, Nencki Institute of Experimental Biology, Polish Academy of Sciences, Warsaw, Poland \\ * Correspondence: halina.gabrys@uj.edu.pl \\ † Present address: The Franciszek Górski Institute of Plant Physiology, Polish Academy of Sciences, Krakow, Poland.
}

Received 29 July 2015; Accepted 11 February 2016

Editor: Markus Teige, University of Vienna

\begin{abstract}
Calcium is involved in the signal transduction pathway from phototropins, the blue light photoreceptor kinases which mediate chloroplast movements. The chloroplast accumulation response in low light is controlled by both phot 1 and phot2, while only phot2 is involved in avoidance movement induced by strong light. Phototropins elevate cytosolic $\mathrm{Ca}^{2+}$ after activation by blue light. In higher plants, both types of chloroplast responses depend on $\mathrm{Ca}^{2+}$, and internal calcium stores seem to be crucial for these processes. Yet, the calcium signatures generated after the perception of blue light by phototropins are not well understood. To characterize the localization of calcium in Arabidopsis mesophyll cells, loosely bound (exchangeable) $\mathrm{Ca}^{2+}$ was precipitated with potassium pyroantimonate and analyzed by transmission electron microscopy followed by energy-dispersive X-ray microanalysis. In dark-adapted wild-type Arabidopsis leaves, calcium precipitates were observed at the cell wall, where they formed spherical structures. After strong blue light irradiation, calcium at the apoplast prevailed, and bigger, multilayer precipitates were found. Spherical calcium precipitates were also detected at the tonoplast. After red light treatment as a control, the precipitates at the cell wall were smaller and less numerous. In the phot2 and phot1phot2 mutants, calcium patterns were different from those of wild-type plants. In both mutants, no elevation of calcium after blue light treatment was observed at the cell periphery (including the cell wall and a fragment of cytoplasm). This result confirms the involvement of phototropin2 in the regulation of $\mathrm{Ca}^{2+}$ homeostasis in mesophyll cells.
\end{abstract}

Key words: Arabidopsis thaliana, blue light, calcium signaling, chloroplast movements, mesophyll cells, phototropin2.

\section{Introduction}

Calcium ions are considered to be an extremely versatile secondary messenger, a key element of many responses to biotic and abiotic factors (for a review, see Dodd et al., 2010). In plants, specific 'calcium signatures' are generated, mainly in the cytosol, but also in the nucleus, mitochondria, and chloroplasts (Stael et al., 2012). The precise control of $\mathrm{Ca}^{2+}$ translocations between organelles and the apoplast generates spatially and temporally distinct cytosolic calcium patterns and thus produces stimulus-specific responses (McAinsh and Pittman, 2009).

In the case of blue light signaling, calcium elevation follows the activation of phototropins. Phototropins are UVA and 
blue light photoreceptor kinases which mediate plant movements and rapid growth responses. The two phototropins of Arabidopsis thaliana, phot 1 and phot2, are characterized by different light sensitivities, though they share highly redundant functions. They both control phototropism, leaf expansion, stomatal opening, and the chloroplast accumulation response. Only phot 1 mediates the inhibition of the hypocotyl growth reaction, and only phot 2 mediates chloroplast avoidance and dark positioning (for a review, see Christie, 2007). Although both Arabidopsis phototropins are responsible for $\mathrm{Ca}^{2+}$ mobilization after blue light treatment, differences in the localization of cellular calcium signals depending on light intensity, plant organ, and the phototropin itself have been reported (Harada and Shimazaki, 2007).

In 10- to 16-day-old Arabidopsis seedlings, a pulse of very strong blue light $\left(10 \mathrm{~s}, 600 \mu \mathrm{mol} \mathrm{m}^{-2} \mathrm{~s}^{-1}\right)$ resulted in a transient increase in cytosolic calcium concentration, $\left[\mathrm{Ca}^{2+}\right]_{\mathrm{cyt}}$, which diminished after treatment with lanthanum $\left(\mathrm{La}^{3+}\right)$ ions (calcium channel inhibitors). In phot 1 mutant seedlings, $\left[\mathrm{Ca}^{2+}\right]_{\mathrm{cyt}}$ was increased in darkness, but the elevation after blue light treatment was half that of the wild type. This indicated the role of phot1 in mobilizing $\mathrm{Ca}^{2+}$ from intercellular spaces through cell membrane-localized channels (Baum et al., 1999). In 3-day-old etiolated Arabidopsis wild-type seedlings, an increase in calcium concentration was observed after a pulse of blue light $\left(10 \mathrm{~s}, 100 \mu \mathrm{mol} \mathrm{m}^{-2} \mathrm{~s}^{-1}\right)$. This response was inhibited by BAPTA ( $\mathrm{Ca}^{2+}$ chelator), similarly to the inhibition of hypocotyl growth, controlled by phot1 (Folta et al., 2003). In 4-day-old etiolated Arabidopsis wild-type seedlings, the illumination of hypocotyls and cotyledons with continuous blue light $\left(25 \mu \mathrm{mol} \mathrm{m}^{-2} \mathrm{~s}^{-1}\right)$ for 10 min caused a transient increase in $\left[\mathrm{Ca}^{2+}\right]_{\text {cyt. }}$. In wild-type and phot 2 mutant plants, the biggest influx of calcium was detected in the third minute after turning on the light. In the phot 1 mutant, a residual effect was present and, since it was not observed at all in the double photlphot 2 mutant, calcium mobilization from the apoplast was attributed to photl (Babourina et al., 2002). A potentialgated cation channel called PACC, a phototropin-activated calcium-permeable channel, was identified in mesophyll protoplasts based on the observation of a $\mathrm{Ca}^{2+}$ flux after continuous illumination with blue light of $275 \mu \mathrm{mol} \mathrm{m}{ }^{-2} \mathrm{~s}^{-1}$. This process became saturated after 11-16 min of irradiation and was inhibited by $\mathrm{La}^{3+}$ and protein kinase inhibitors. In the phot 1 mutant, the activity of this channel was greatly reduced and it was not detected in the double photlphot 2 mutant (Stoelzle et al., 2003).

In 3-week-old Arabidopsis rosette leaves, changes in $\left[\mathrm{Ca}^{2+}\right]_{\text {cyt }}$ were observed after $10 \mathrm{~s}$ blue light pulses. In wildtype plants, an increase in calcium concentration occurred at light intensities of $0.1-250 \mu \mathrm{mol} \mathrm{m}{ }^{-2} \mathrm{~s}^{-1}$, phot1 being active in the range of $0.1-50 \mu \mathrm{mol} \mathrm{m}^{-2} \mathrm{~s}^{-1}$ and phot 2 in the range of 1-250 $\mu \mathrm{mol} \mathrm{m}{ }^{-2} \mathrm{~s}^{-1}$. Calcium channel inhibitors, $\mathrm{Co}^{2+}, \mathrm{La}^{3+}$, and nifedipine, and calcium chelators, EGTA and BAPTA, caused a significant reduction in the blue light response in wild-type plants and phototropin mutants, indicating a mechanism of $\mathrm{Ca}^{2+}$ influx into the cytoplasm through membrane channels regulated by both photoreceptors. On the other hand, phospholipase C inhibitors neomycin and U-73122 inhibited the blue light increase in $\left[\mathrm{Ca}^{2+}\right]_{\text {cyt }}$ in wild-type and phot 1 mutant plants, but not in the phot 2 mutant. Thus phot2 was suggested to be responsible for the phospholipase $\mathrm{C}$-dependent release of $\mathrm{Ca}^{2+}$ from internal stores, such as the vacuole or endoplasmic reticulum (ER) (Harada et al., 2003).

The role of calcium in the control of chloroplast movements was proposed long before the discovery of phototropins (for a review, see Banaś et al., 2012). The rotation of the flat, ribbon-like chloroplast of the filamentous alga Mougeotia was shown to depend on the presence of specialized membrane vesicles which contain calcium ions (Wagner and Klein, 1981). However, other works using calcium inhibitors suggested that the disruptions in chloroplast rotation resulted from toxic effects or disturbances in processes other than the photosensory transduction chain (Schönbohm et al., 1990). In Vallisneria gigantea, the movement of chloroplasts under red light correlated with cytoplasmic streaming within the cell and was precisely regulated by $\mathrm{Ca}^{2+}$ concentration (Takagi and Nagai, 1983). In Physcomitrella patens (Sato et al., 2003) and Adiantum capillus-veneris (Sato et al., 2001), external calcium was involved in triggering chloroplast movement induced by mechanical stimulation. Inhibitors of calcium channels $\left(\mathrm{La}^{3+}\right.$ and $\mathrm{Ga}^{3+}$ ) did not affect the photorelocation of chloroplasts in these species.

Two types of blue light-controlled chloroplast movements have been characterized in higher plants. One is the accumulation response observed in Arabidopsis under low blue light (0.08-4 $\left.\mu \mathrm{mol} \mathrm{m}{ }^{-2} \mathrm{~s}^{-1}\right)$. Chloroplasts move to cell walls lying perpendicular to the direction of incident light. The other is the avoidance response. Under strong blue light $\left(>20 \mu \mathrm{mol} \mathrm{m}^{-2} \mathrm{~s}^{-1}\right)$, chloroplasts gather under walls parallel to the direction of incident light (Trojan and Gabryś, 1996; Sakai et al., 2001).

The involvement of calcium in the control of blue lightinduced chloroplast relocations was put forward on the basis of inhibitor treatments in the aquatic angiosperm Lemna trisulca (Tlałka and Gabrys, 1993; Tlałka and Fricker, 1999). A prolonged incubation (12h) with EGTA was needed for a partial inhibition of chloroplast avoidance. However, in tissues initially pre-treated with the A23187 ionophore, only $1 \mathrm{~h}$ long EGTA incubation was sufficient to disturb chloroplast movements. Similarly, A23187 pre-treatment followed by $1 \mathrm{~h}$ incubation with lanthanum enhanced its inhibitory effect on chloroplast relocations. These observations indicated the key role of internal calcium stores in the control of chloroplast movements (Tlałka and Gabrys, 1993). This hypothesis was supported by strong inhibitory effects observed after $2 \mathrm{~min}$ treatments with caffeine (which causes a release of calcium from intracellular stores) and thapsigargin (a selective inhibitor of the ER $\mathrm{Ca}^{2+}$-ATPase). The incubation of Lemna fronds with calcium channel inhibitors nifedipine (for $3 \mathrm{~h}$ ) and verapamil (for $1 \mathrm{~h}$ ) also partially disturbed chloroplast movements (Tlałka and Fricker, 1999).

In Nicotiana tabacum, the exogenous application of $\mathrm{Ca}^{2+}$ did not affect movements. However, the disturbance in calcium homeostasis by incubating the tissue with the A23187 ionophore proved detrimental to chloroplast relocations. In tobacco, in contrast to duckweed, short-term treatment with EGTA or TFP (trifluoperazine; an inhibitor of calmodulin) 
was sufficient to inhibit both chloroplast responses strongly. This effect was at least partially caused by disorders in the actin cytoskeleton, which is indispensable for movements. The subsequent addition of $\mathrm{Ca}^{2+}$ partially restored the ability of chloroplasts to move (Anielska-Mazur et al., 2009). A similar movement reactivation after EGTA treatment was observed in Adiantum (Kadota and Wada, 1992). Inhibition by TFP implicated a calmodulin-dependent signal transduction pathway, consistent with previous findings on chloroplast rotation control in Mougeotia (Wagner et al., 1984).

Indirect evidence for the role of calcium in the control of chloroplast relocations in Arabidopsis came from a study showing the involvement of phosphoinositides in the blue light signaling pathway. The phospholipase $\mathrm{C}$ pathway was suggested to take part in phot2 signaling, while the phosphatidylinositol kinases PI3K and PI4K were suggested to control the accumulation response mediated by both phototropins. U73-122 (the phospholipase C inhibitor) as well as wortmannin and LY294002 (two inhibitors of the PI3K pathway) suppressed the transient calcium elevation induced by blue light in Arabidopsis leaves (Aggarwal et al., 2013a, b). In line with that, the inhibitory effect of wortmannin on chloroplast movements in Nicotiana could be over-ridden by the application of external $\mathrm{Ca}^{2+}$ (Anielska-Mazur et al., 2009).

Even though substantial evidence has been collected, the role of calcium in phototropin-controlled physiological responses needs further investigation. $\mathrm{Ca}^{2+}$ fluxes have been analyzed by aequorin luminescence (Baum et al., 1999; Harada et al., 2003), ion-selective microelectrode (Babourina et al., 2002), and patch-clamp methods (Stoelzle et al., 2003). These show changes in calcium concentration mainly as a function of time. Although indirect evidence has been obtained using inhibitors, differences in the spatial distribution of $\mathrm{Ca}^{2+}$ within the cell and the relevant calcium storage compartments remain to be specified. Studies on calcium in Arabidopsis leaves (Harada et al., 2003) and mesophyll protoplasts (Stoelzle et al., 2003) determine light ranges in which phot 1 and phot 2 affect $\mathrm{Ca}^{2+}$ concentrations; however, they do not answer the question of how these changes correspond to chloroplast movement signaling. Spatial and temporal aspects of calcium release are important to understand how specific calcium signatures are generated. In this work, $\mathrm{Ca}^{2+}$ precipitation with KPA (potassium pyroantimonate) and transmission electron microscopy (TEM) followed by X-ray microanalysis were employed to study changes in calcium distribution. The main aim was to determine the localization of $\mathrm{Ca}^{2+}$ after blue light treatment in Arabidopsis mesophyll cells in order to elucidate how calcium participates in directing chloroplast movements. The intensity of blue light and the duration of irradiation were chosen to investigate the phot2specific chloroplast avoidance response.

\section{Materials and methods}

\section{Plant material and growth conditions}

Seeds of Arabidopsis wild-type Columbia were obtained from Nottingham Arabidopsis Stock Centre (Nottingham, UK). Mutant seeds were the kind gifts of A.R. Cashmore, the Plant Science
Institute, Department of Biology, University of Pennsylvania, Philadelphia, USA (phot2) and J. Jarillo, Instituto Nacional de Investigación y Tecnología Agraria y Alimentaria, Madrid, Spain (phot1phot2). Plants were grown in a growth chamber (Sanyo MLR $350 \mathrm{H}$, Japan) with a $10 \mathrm{~h} / 14 \mathrm{~h}$ light/dark cycle at $23^{\circ} \mathrm{C}$, with $80 \%$ relative humidity, and illuminated by fluorescent lamps (FL40SS.W/37, Sanyo, Japan) with a photosynthetic photon flux density of $60-100 \mu \mathrm{mol} \mathrm{m}{ }^{-2} \mathrm{~s}^{-1}$.

\section{Tissue processing}

Five-week-old plants were dark-adapted for at least $12 \mathrm{~h}$. The fifth or sixth rosette leaf was irradiated directly on the plant for 3 min with blue light (LXHL-PR09, Ledium Ltd, Hungary) of $100 \mu \mathrm{mol} \mathrm{m} \mathrm{m}^{-2}$ $\mathrm{s}^{-1}$ to induce the phot2-mediated chloroplast avoidance response. Control plants were kept untreated in darkness or were irradiated for $3 \mathrm{~min}$ with equimolar red light (LXHL-PD09, Ledium Ltd), which does not activate phototropins. Immediately after treatment, leaves were cut into $2 \mathrm{~mm}$ strips and put directly into the fixative solution. A suitable cutting margin was taken into account, so that the wounded site did not lie adjacent to the cells used for analysis. In order to minimize calcium elution, pyroantimonate precipitation was performed concomitantly with material fixing. Tissue sections were infiltrated with a syringe containing a fixative solution of $2 \%$ glutaraldehyde, $2 \%$ potassium pyroantimonate in a phosphate buffer $\left(100 \mathrm{mM} \mathrm{KH} \mathrm{PO}_{4} / \mathrm{K}_{2} \mathrm{HPO}_{4}, \mathrm{pH} 7.4\right)$ at $4{ }^{\circ} \mathrm{C}$ (according to Tretyn et al., 1992; Musetti and Favali, 2003) and incubated on ice for $2 \mathrm{~h}$. All steps were performed in darkness, using only 'safe' green light. After fixation, the material was washed four times in a chilled phosphate buffer $\left(100 \mathrm{mM} \mathrm{KH} \mathrm{PO}_{4} / \mathrm{K}_{2} \mathrm{HPO}_{4}, \mathrm{pH} 7.6,3 \times 10 \mathrm{~min}\right.$, $1 \times 15 \mathrm{~min}$ ) and subsequently stained with $1 \%$ osmium tetra-oxide in a $100 \mathrm{mM}$ phosphate buffer at $4{ }^{\circ} \mathrm{C}$. After dehydration in an acetone series $(10,30,50,70,90,96,100 \%, 2 \times 5 \mathrm{~min})$, the material was embedded in epoxy resin of low viscosity (Spurr, 1969). Crosssections of leaves $(100 \mathrm{~nm}$ thick) were obtained with an EM-U-C6 ultramicrotome (Leica, Austria) and put on copper grids coated with formvar and carbon.

\section{TEM and X-ray microanalysis}

Leaves were harvested from at least two (2-4) different batches of plants. Samples were analyzed under a JEM 1400 transmission electron microscope (JEOL Co., Japan) equipped with a Morada CCD camera (SiS-Olympus, Japan) at an accelerating voltage of $80 \mathrm{keV}$. The X-ray microanalysis was performed using the energy-dispersive full range X-ray microanalysis system EDS INCA Energy TEM (Oxford Instruments, UK). The detection and semi-quantitative analysis of calcium and antimony were carried out by collecting $\mathrm{X}$-ray spectra from a selected region of interest in the energy range of $1-10 \mathrm{keV}$ (Fig. 1). The relative content of calcium and antimony was calculated using standards on the basis of the peak area characteristic of the calcium- $\mathrm{K}_{\alpha}$ emission line $(3.691 \mathrm{keV})$ and antimony$\mathrm{L}_{\alpha}$ emission line (3.605 keV), with Oxford INCA TEM 200 software. The identification and localization of calcium precipitates in cells was determined by mapping the distribution of the element in certain parts of the tissue (X-ray mapping). The semi-quantitative analysis was carried out by measuring the spectra from identical squares $\left(10 \mu^{2}\right)$ for $200 \mathrm{~s}$, at $\times 15000$ magnification, from the peripheral areas of mesophyll cells including the cell wall and a fragment of cytoplasm (Fig. 1). The regions inside the vacuole and intercellular space, which rarely contained visible precipitates, were chosen as controls. The data on content of elements were processed using EDS INCA software for the assessment of the percentage weight concentration of elements after correction for interelement effects $(\mathrm{Wt} \%)$. Spectra were recorded in at least five (5-9) different regions of the leaf mesophyll. A 3D model of precipitates was created with a tomographic holder and Chimera software. The surface area for precipitate cross-sections was measured with the particle analysis procedure available in ImageJ. Particle analysis was performed on 


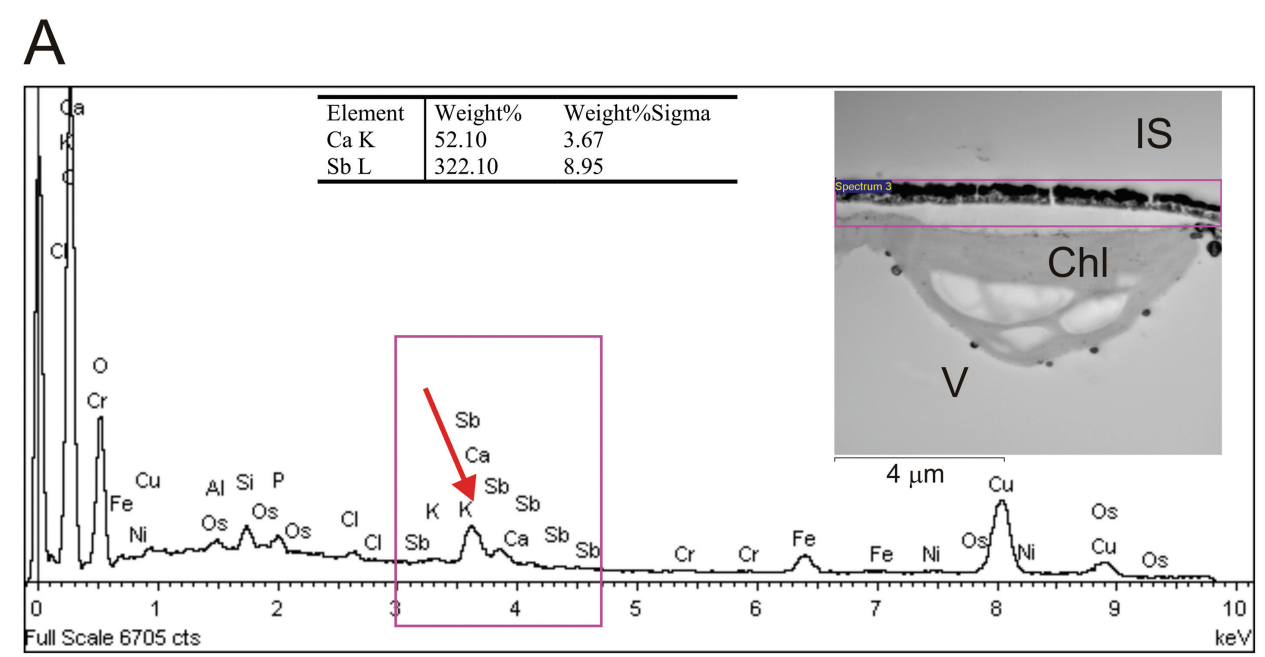

B

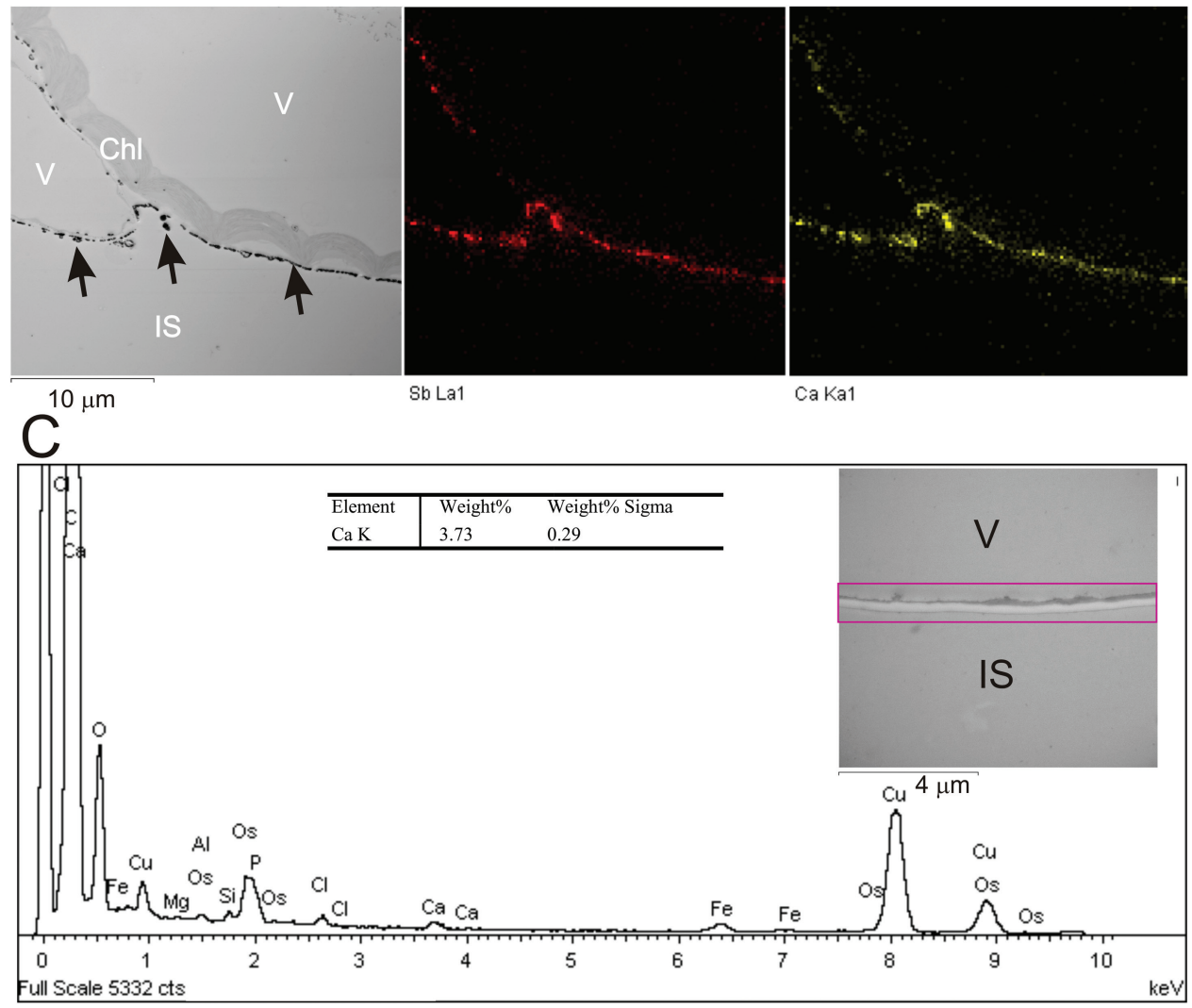

Fig. 1. (A) Example X-ray spectra obtained from a selected rectangular region of the periphery of an Arabidopsis wild-type mesophyll cell (including the cell wall and a fragment of cytoplasm), which contains precipitates of calcium and antimony. The relative content (Weight \%) of individual elements was calculated on the basis of the peak area (arrow) characteristic of the calcium-Ka line (Ca K) and antimony-La line (Sb $L)$. The error value quoted is sigma, which is the statistical error for the calculated Wt\%. (B) An example map showing calcium (yellow) and antimony (red) localization within the analyzed cells. (C) Control X-ray spectra obtained from a selected rectangular region of the periphery of a mesophyll cell (including the cell wall and a fragment of cytoplasm) not treated with potassium pyroantimonate. Chl, chloroplast; IS, intercellular space; V, vacuole.

images showing leaf mesophyll (magnifications between $\times 400$ and $\times 1020$ ), which were manually segmented into cell regions (cell wall, vacuole, and tonoplast). The surface area of precipitates adjacent to the outer face of the cell wall was expressed per unit of the cell wall length, while the area of precipitates localized in the vacuole at the tonoplast was expressed per unit of the tonoplast length. The significance of the effects of light conditions and the plant line on the mean calcium content and precipitate area was assessed with ANOVA. For pairwise comparison of means, Tukey's test was performed after one-way ANOVA, calculated separately for each plant line. Adjusted $P$-values from Tukey's test are indicated in the figures with asterisks. The tests were performed with R-software.

\section{Results}

The KPA precipitation method does not depict the overall distribution of calcium within cells, but only that fraction which is susceptible to precipitation. Any calcium present in 
an appropriate concentration, not too tightly bound to cellular organelles and not readily washed out during the procedure, may be analyzed. The analysis of KPA precipitates yields information about the difference in the distribution and content of calcium dependent on a given factor, so the interpretation of results should be done on the basis of controls. In our experimental model, Arabidopsis leaves were exposed to blue light of $100 \mu \mathrm{mol} \mathrm{m} \mathrm{m}^{-2} \mathrm{~s}^{-1}$ for $3 \mathrm{~min}$ to activate phot2 and induce a measurable chloroplast avoidance response. In wild-type plants, this time point corresponds to the moment when chloroplasts achieve maximum velocity after the onset of strong blue light (Labuz et al., 2015). Control leaves were incubated in the dark or irradiated with equimolar red light which does not affect phototropins. Carefully selected conditions, including the use of a phosphate buffer with a slightly alkaline $\mathrm{pH}$ along with glutaraldehyde fixation, were optimized for the formation of pyroantimonate precipitates only with calcium ions, while reducing the specificity of the reaction to monovalent ions and $\mathrm{Mg}^{2+}$ (Wick and Hepler, 1982). Spectra (Fig. 1A) and maps (Fig. 1B) of relevant cell areas were collected in order to confirm the co-localization of calcium (marked yellow) with antimony (marked red) in these electron-dense structures. A control spectrum showing the element composition in cells treated without KPA is shown in Fig. 1C.

\section{The localization of calcium in the mesophyll cells of wild-type Arabidopsis}

In the mesophyll cells of dark-adapted Arabidopsis wild-type leaves, calcium precipitates formed circular, semi-circular, or lenticular structures of different sizes (Fig. 2A-D). At the cell wall these spherical structures had several layers concentrically propagating in both directions, from and into the cell
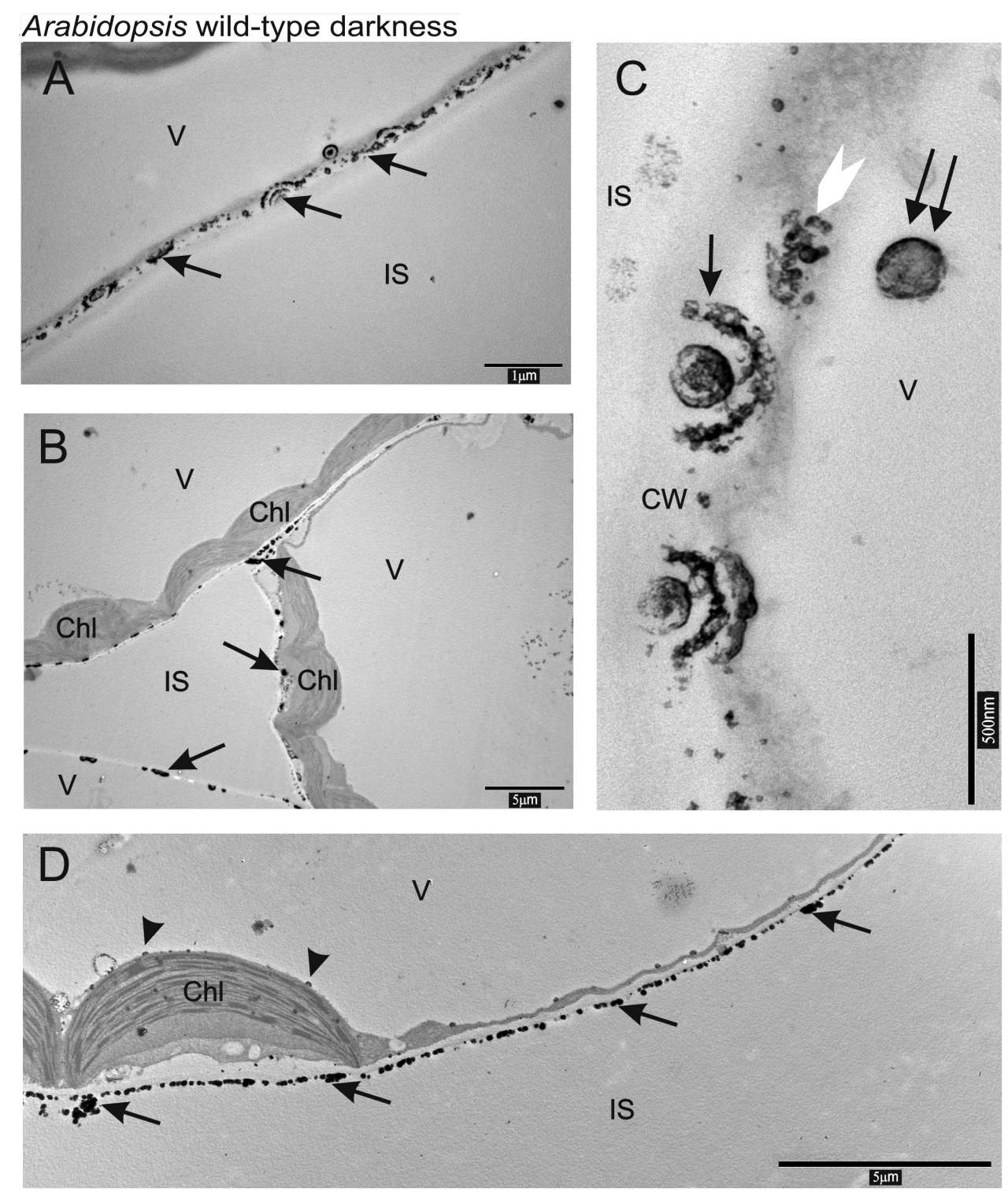

Fig. 2. (A-D) The localization of calcium and antimony precipitates in Arabidopsis wild-type mesophyll cells in darkness. Arrows indicate precipitates of calcium and antimony at the cell wall; the white arrowhead precipitates in the cytosol; a double arrow precipitates in the vacuole; and black arrowheads precipitates adjacent to the chloroplast envelope and the tonoplast. CW, cell wall, Chl, chloroplast; IS, intercellular space; V, vacuole. 
(Fig. 2A, C). Precipitates of higher densities were observed along the cell walls (Fig. 2D). Circular precipitates and very small granules were occasionally found in the cytosol, in the vacuole (Fig. 2C), and at the chloroplast envelope outer membrane (Fig. 2D).

In cells irradiated with blue light for $3 \mathrm{~min}$, the calcium localization pattern was similar to that in darkness. However, the size and density of structures in the regions of cell walls facing the intercellular spaces were significantly increased (Fig. 3A, D). There were relatively few precipitates in the middle lamella (Fig. 3B). Single (Fig. 3C, F) and clustered (Fig. 3A, D) structures were found along the edges of cell walls. Some of these were surrounded by dark gray or black bands of varying thickness arranged in several concentric layers (Fig. 3C, D). A typical picture of cells after blue light treatment showing the connected precipitates which align along the cell wall is shown in Fig. 3A. Analysis of cell wall cross-sections revealed that calcium precipitates were formed by overlapping hemispheres (Fig. 3F). This observation was confirmed by a 3D model based on tomography images. The 3D structure was limited by the thickness of the slice $(100 \mathrm{~nm})$, but it demonstrated that cross-sections indeed represent the segments of a sphere (Fig. 3D, E). In areas where precipitates were rarely seen in dark conditions, such as the cytosol (Fig. 3B, white arrow), tonoplast, and the chloroplast envelope (Fig. 3A), they became more abundant after blue light treatment (Fig. 3A, B, D). Semicircular or lenticular precipitates on the tonoplast were oriented towards the vacuole interior (Fig. 3A, long white arrow) and those observed on the outer membrane of the chloroplast envelope pointed towards the cytosol (Fig. 3A short white arrow).
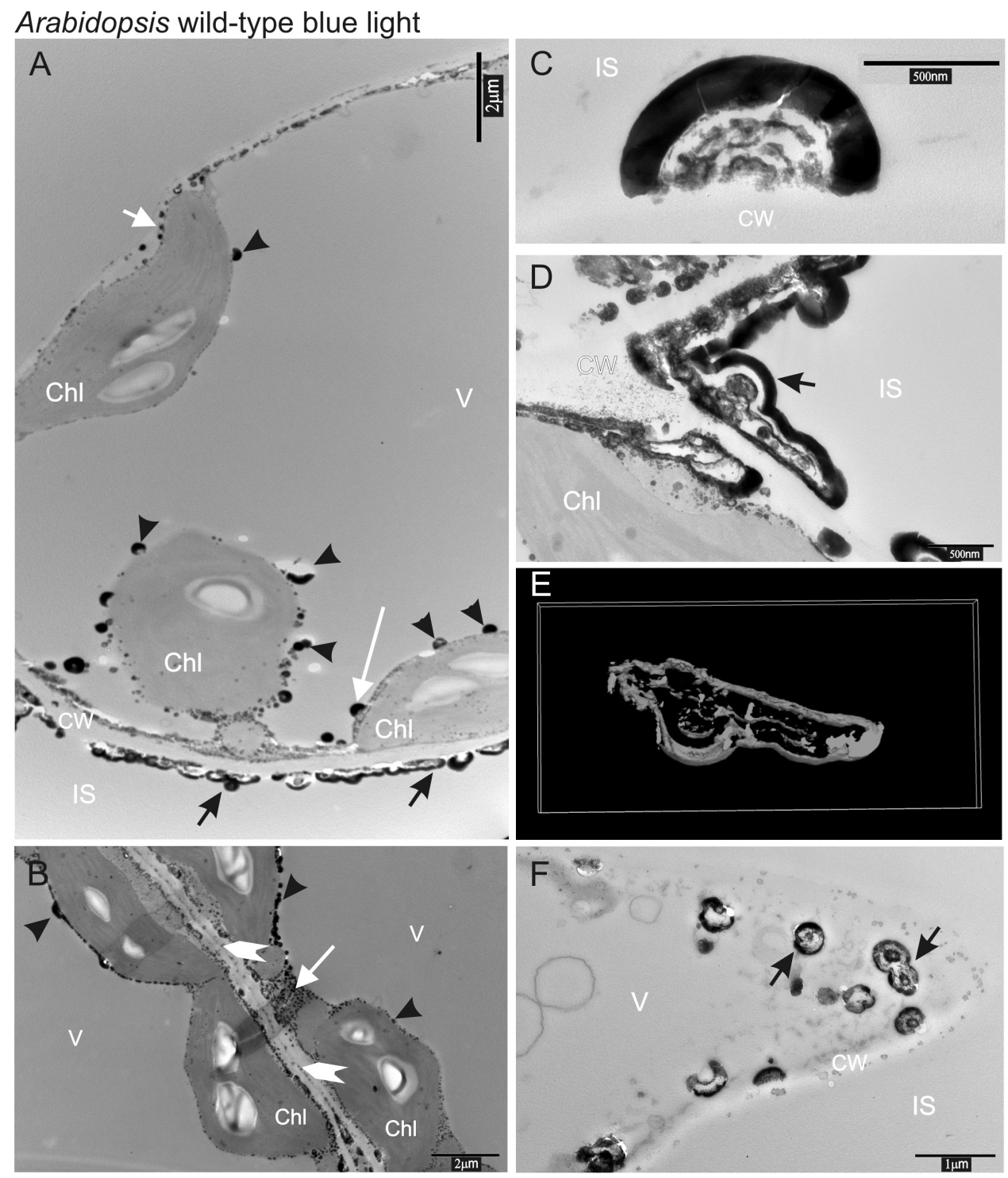

Fig. 3. (A, B) The localization of calcium and antimony precipitates in Arabidopsis wild-type mesophyll cells after 3 min blue light treatment of $100 \mu \mathrm{mol} \mathrm{m} \mathrm{m}^{-2} \mathrm{~s}^{-1}$. Cross-section of the precipitates (C, D) perpendicular and (F) parallel to the cell wall plane. (E) The 3D model of the precipitate created from (D). Arrows indicate precipitates of calcium and antimony at the cell wall; white arrowheads precipitates in the middle lamella; and black arrowheads precipitates adjacent to the chloroplast envelope and the tonoplast. A long white arrow indicates precipitates on the tonoplast directed towards the vacuole interior; a short white arrow precipitates on the outer membrane of the chloroplast envelope pointing towards the cytosol; and a medium sized arrow indicates precipitates in the cytosol. CW, cell wall, Chl, chloroplast; IS, intercellular space; V, vacuole. 
After 3 min of red light treatment, the precipitates at the cell wall were much smaller and did not form the characteristic spherical structures (Fig. 4A-D) as compared with darkness and blue light. No precipitates were observed in the cytosol, on the tonoplast, and on the outer membrane of the chloroplast envelope (Fig. 4A, B).

\section{The localization of calcium in the mesophyll cells of Arabidopsis phototropin mutants}

In order to link the differences in $\mathrm{Ca}^{2+}$ patterns observed after light treatments with phot 2 signaling, the localization of calcium precipitates in the phot 2 and phot1phot 2 mutants was investigated. Only phot 2 is responsible for calcium mobilization in mature Arabidopsis leaves under the light conditions used in this study $\left(100 \mu \mathrm{mol} \mathrm{m} \mathrm{m}^{-2} \mathrm{~s}^{-1}\right)$, as demonstrated by Harada et al. (2003). Figure 5 shows the localization of calcium precipitates in the cells of the phototropin mutants. Generally, a smaller variation in calcium structures at the cell wall was observed in these mutants. Calcium precipitates forming spherical structures were rarely multilayered. In dark conditions, the phot 2 and phot1phot 2 mutants had precipitates with a firm 'bead' structure, which usually merged into bands running along the cell walls, as compared with the Arabidopsis wild type (compare Figs 2 and 5 phot 2 darkness, phot1phot2 darkness). In the phot 2 mutant, structures at the cell wall under blue light and red light were similar to those observed under dark conditions. Precipitates found in phot 2 after red light had a defined spherical structure, in contrast to those observed in wild-type plants (compare Figs 4 and 5 phot 2 red light). Round precipitates localized in the cytosol, at the chloroplast envelope, and the tonoplast were frequently found in the phot 2 mutant, regardless of the experimental conditions. In the double phototropin mutant, calcium precipitates after blue light were comparable with those in darkness. After red light treatment, they were much smaller and resembled those observed in wild-type plants.

\section{Calcium content and the area of precipitates in Arabidopsis mesophyll cells}

To quantify the effects of light observed in the phototropin mutants, the calcium content and the area of precipitates were measured. The calcium content was determined semiquantitatively, based on X-ray microanalysis spectra. The spectra from rectangular areas of the same size covered the periphery of a mesophyll cell (including the cell wall and a fragment of cytoplasm) lying along the cell wall (Fig. 1A). For each spectrum on the cell periphery, two control spectra were measured in regions of the vacuole and intercellular spaces. Usually small amounts of calcium were found in these control areas (results not shown). The lowest calcium content at the cell wall was in wild-type plants in darkness (Fig. 6A). Following blue light treatment, the calcium content was considerably higher than in dark conditions. This effect was also observed after red light, but was less prominent. In

\section{Arabidopsis wild-type red light}
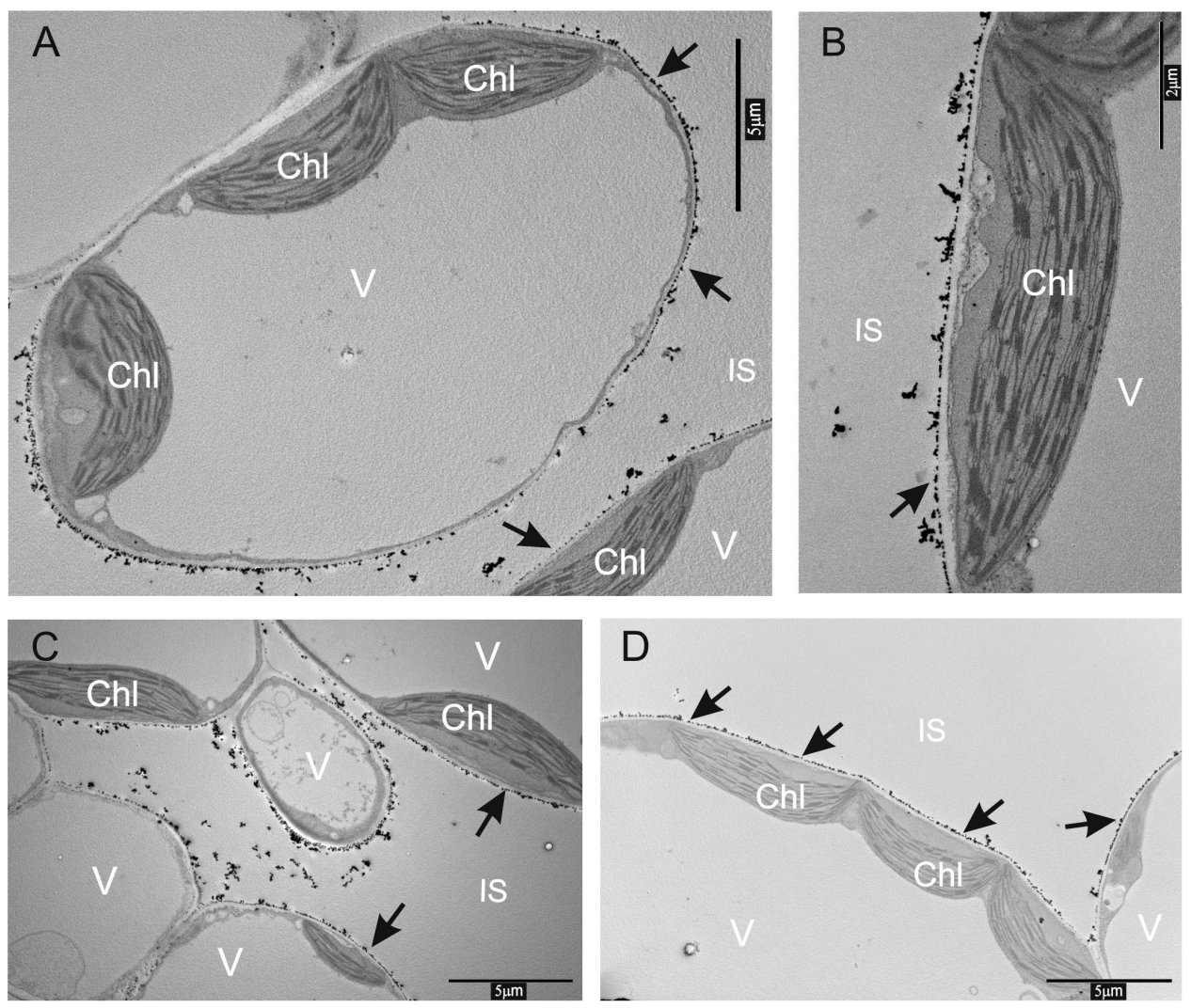

Fig. 4. (A-D) The localization of calcium and antimony precipitates in Arabidopsis wild-type mesophyll cells after 3 min red light treatment of $100 \mu \mathrm{mol} \mathrm{m} \mathrm{m}^{-2} \mathrm{~s}^{-1}$. Arrows indicate precipitates of calcium and antimony at the cell wall. Chl, chloroplast; IS, intercellular space; V, vacuole. 
phot2 darkness

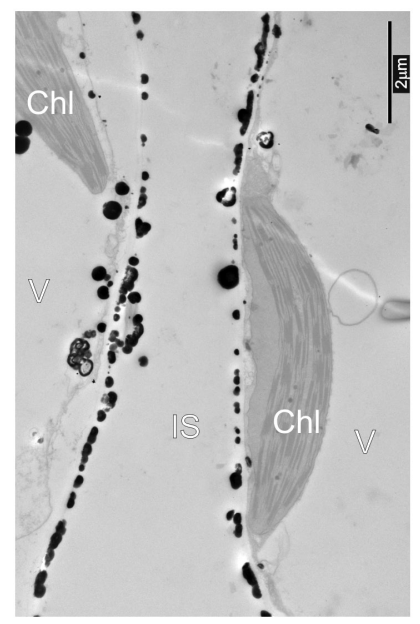

phot1phot2 darkness

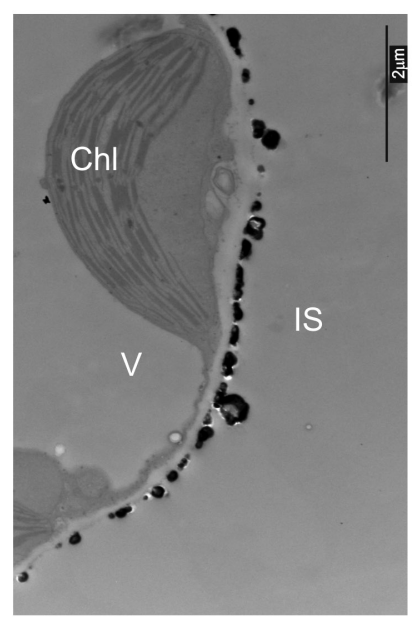

phot2 blue light

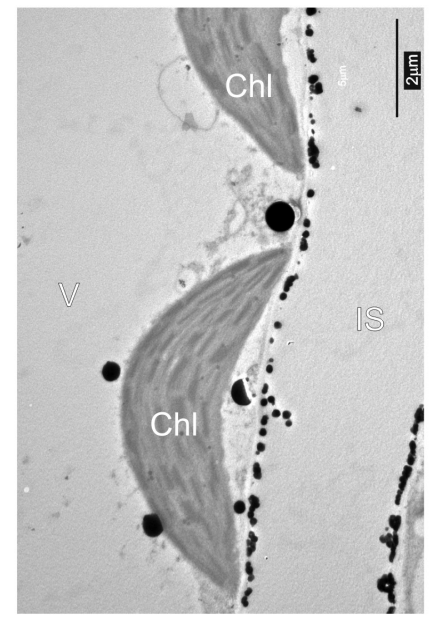

phot1phot2 blue light

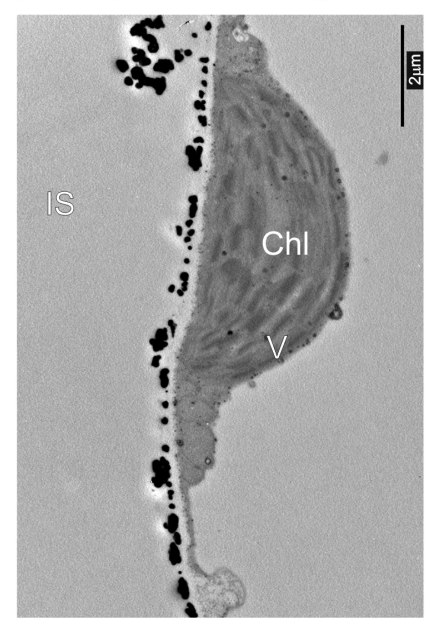

phot2 red light

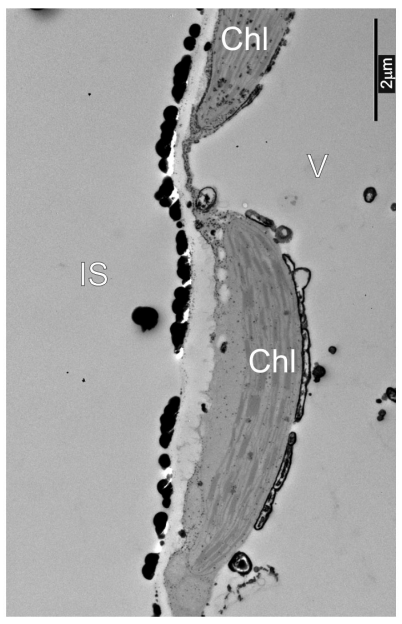

phot1phot2 red light

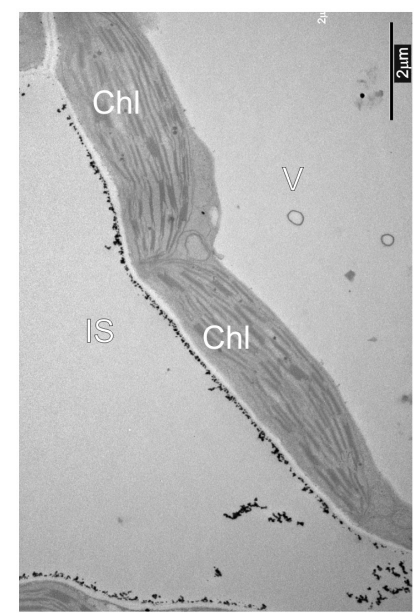

Fig. 5. The localization of calcium and antimony precipitates in mesophyll cells of the Arabidopsis phototropin mutants phot2 and phot1phot2 in darkness, after $3 \mathrm{~min}$ of blue or red light treatment of $100 \mu \mathrm{mol} \mathrm{m}{ }^{-2} \mathrm{~s}^{-1}$. Chl, chloroplast; IS, intercellular space; V, vacuole.

darkness the phot 2 and photlphot 2 mutants had a higher calcium content in the analyzed regions than wild-type plants. In the phot 2 mutant, the amount of calcium increased only slightly after blue light treatment as compared with darkness, but it increased strongly after red light irradiation. The calcium content in the double phototropin mutant did not change significantly after blue light, as compared with darkness. Red light treatment decreased the content of calcium in this mutant, but this effect was not statistically significant. Changes in the antimony content in precipitates at the cell wall reflected those observed for calcium in all experimental groups (Fig. 6B).

To quantify the precipitates in cell wall regions facing the intercellular space, their surface area was calculated and normalized to the cell wall length (Fig. 7A). Only the precipitates seen in contact with the cell wall were taken into account. In wild-type plants the precipitate area was larger after blue light treatment as compared with dark-adapted and red lighttreated plants. The phot 2 mutant exhibited larger precipitates after red light treatment, while the difference between darkness and blue light was insignificant. In the phototropin double mutant, light conditions did not influence the area of precipitates. In all light conditions and plant lines, the precipitates in the vacuole were located mainly at the tonoplast. Their surface area was expressed per unit of tonoplast length. In wild-type plants, the surface area was significantly higher in blue light-irradiated than in dark-adapted leaves (Fig. 7B). No precipitates were observed in red light-irradiated samples. In phot 2 and the double mutant, the precipitate area was not significantly affected by light conditions.

\section{Discussion}

Calcium imaging (for a review, see Batistic and Kudla, 2012) in mesophyll cells after light treatment presents a number of difficulties. A major obstacle to the use of fluorescence microscopy lies in the autofluorescence of cell components (Miyawaki et al., 1999). The introduction of fluorescent dyes is inefficient and results in toxic effects which hamper chloroplast movements, as shown in Lemna (Tlałka and Fricker, 1999). The cameleon system, which relies on the sensitivity of calmodulin to calcium and on the resonance energy transfer between different green fluorescent protein (GFP) variants, is difficult to handle because the excitation wavelength 
$\mathrm{Ca}$

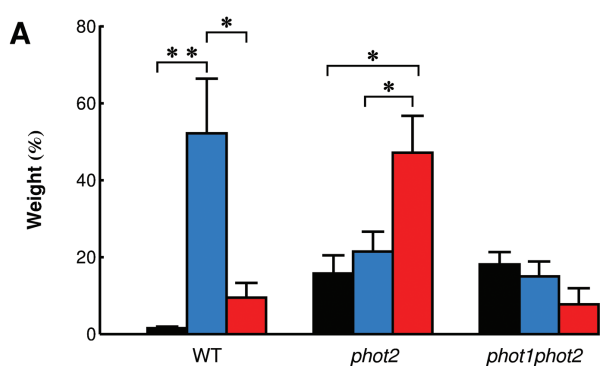

$\mathrm{Sb}$

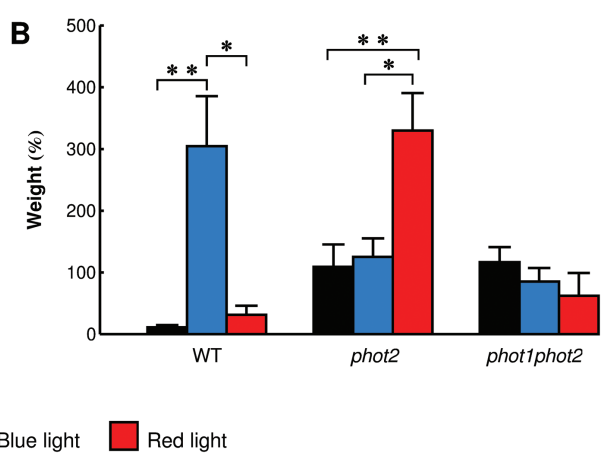

Fig. 6. The relative content of (A) calcium and (B) antimony in precipitates at the periphery of mesophyll cells (including the cell wall and a fragment of cytoplasm) of the Arabidopsis wild type and phot2, phot1phot2 mutants. The relative content of a given element in precipitates from dark-adapted leaves (black bars), leaves after blue light irradiation (blue bars), and leaves after red light irradiation (red bars). Each bar shows an average of 5-9 spectra of cell regions obtained from leaves harvested from $2-4$ independent plant batches. Error bars indicate the SE. ${ }^{\star} P=0.01-0.05 ;{ }^{* \star} P=0.001-0.01 ;{ }^{* \star} P<0.001$.

Cell wall

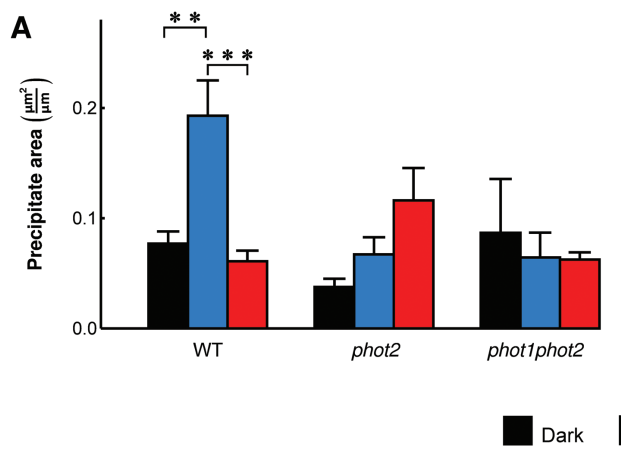

B

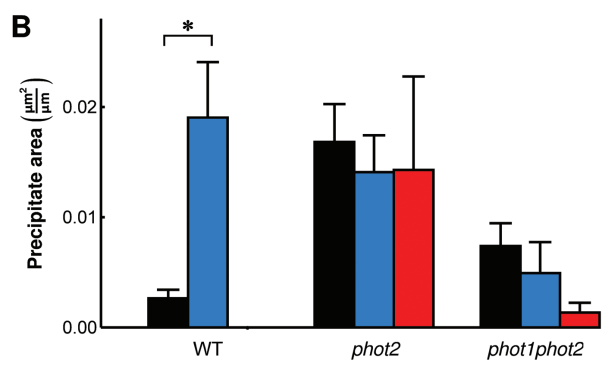

Blue light

Red light

Fig. 7. The surface area of precipitate cross-sections outside the cell walls (A) facing the intercellular space or (B) localized in the vacuole at the tonoplast in wild-type, phot2, and phot1phot2 mutant Arabidopsis mesophyll cells. The area was measured in dark-adapted leaves (black bars), blue lightirradiated leaves (blue bars), and red light-irradiated leaves (red bars). Each bar shows an average of at least five images of mesophyll obtained from leaves harvested from 2-4 independent plant batches. In (B), the bar for the wild-type red light group is missing as no precipitates at the tonoplast were observed in these conditions. Error bars indicate the SE. ${ }^{\star} P=0.01-0.05 ;{ }^{\star \star} P=0.001-0.01 ;{ }^{\star \star \star} P<0.001$.

overlaps with the action spectra of phototropins (Miyawaki et al., 1999). The use of the aequorin system is also limited, as the detection method allows the measurement of calcium changes only after light pulses (Baum et al., 1999; Harada et al., 2003) and provides no direct image of calcium distribution in the cell. The KPA precipitation method chosen in this work identifies a $\mathrm{Ca}^{2+}$ fraction which is relatively loosely bound to cell components and visualizes the dynamics of these mobile ions after a chosen stimulus (Wick and Helper, 1982). The major disadvantage of KPA precipitation is that it does not allow changes in calcium concentration to be continuously monitored. The time resolution of the method is limited by the speed of KPA and fixative penetration in the tissue; thus, real-time investigation of calcium signaling during the onset of strong blue light is not possible. However, the combination of $\mathrm{X}$ - ray analysis with TEM yields more detailed information about calcium localization thanks to its higher resolution as compared with methods based on optical microscopy. In this work, the presence of calcium precipitates within the cell walls, for example in the middle lamella and at the border of the intercellular space or protoplast, was shown very precisely. The morphology of precipitates was also determined and it was confirmed that they indeed contain $\mathrm{Ca}^{2+}$. This would be virtually impossible using fluorescence techniques.

\section{Blue light-dependent calcium localization in Arabidopsis mesophyll cells}

In wild-type Arabidopsis mesophyll cells in darkness, concentric calcium precipitates localized predominantly at the cell wall. Some were also found in the cytoplasm. Their positioning suggests that the flow of calcium ions takes place in both directions, both into and out of the cytoplasm. After $3 \mathrm{~min}$ of blue light irradiation $\left(100 \mu \mathrm{mol} \mathrm{m}^{-2} \mathrm{~s}^{-1}\right)$, spherical structures at the cell wall were bigger, multilayered, and aligned in clusters. Precipitates in the cytoplasm and adjacent to the chloroplast envelope or the tonoplast were also observed. These results demonstrate that blue light causes specific calcium mobilization within the Arabidopsis mesophyll cells. However, the nature of changes in loosely bound calcium content remains obscure. The activity of membrane channels in mesophyll protoplasts after $15 \mathrm{~min}$ of blue light (Stoelzle et al., 2003) and changes in $\left[\mathrm{Ca}^{2+}\right]_{\text {cyt }}$ after a $10 \mathrm{~s}$ blue light pulse (Harada 
et al., 2003) were examined in Arabidopsis leaves. In continuous blue light of a considerably lower intensity $\left(25 \mu \mathrm{mol} \mathrm{m}{ }^{-2}\right.$ $\left.\mathrm{s}^{-1}\right), \mathrm{Ca}^{2+}$ fluxes were analyzed using an ion-selective microelectrode only in decapitated Arabidopsis hypocotyls. In these conditions, the maximum calcium influx into the cell occurred 3 min after irradiation, then calcium efflux began (Babourina et al., 2002). The increase in the number of precipitates at the cell wall and the tonoplast may reflect $\mathrm{Ca}^{2+}$ efflux from the cytoplasm. The alignment of spherical structures which started to connect to each other after blue light irradiation supports this assumption. This efflux may be a part of calcium signal dissipation, a mechanism through which the cell sensitivity to light is sustained during constant illumination. This hypothesis is also consistent with previous results obtained on Lemna cells stained with Fluo-3 as a calcium indicator. Continuous, strong blue light $\left(75.2 \mu \mathrm{mol} \mathrm{m}^{-2} \mathrm{~s}^{-1}\right)$ induced an increase in Fluo-3 fluorescence in the second minute, followed by a decrease in intensity, reaching its initial value after $10 \mathrm{~min}$ (Tlałka and Fricker, 1999).

The circular precipitates and granules observed in the cytoplasm imply the presence of vesicles rich in calcium. As blue light induces their formation, it may be speculated that a fraction of calcium undergoes exo/endocytosis during phototropin signaling. Calcium in multivesicular compartments plays a signaling role during the response to pathogens (An et al., 2006). $\mathrm{Ca}^{2+}$-containing vesicles co-localized in the proximity of the Mougeotia chloroplast. This calcium store was suggested to be important for efficient chloroplast rotation (Wagner and Rossbacher, 1980; Rossbacher and Wagner, 1984). Interestingly, phot2 which is bound to the plasma membrane moves from the cytoplasm into the Golgi complex and post-Golgi structures after blue light treatment (Aggarwal et al., 2014). The physiological role of this receptor trafficking remains unknown, but it might imply that phototropin is involved in formation of the calcium signature in the cytoplasm.

After equimolar red light irradiation, calcium precipitates at the cell wall were smaller and lacked the multilayered structure, but their calcium content measured near the cell wall was elevated as compared with darkness. Our results are in line with the findings of Harada et al. (2003) regarding a red light-induced transient increase in $\left[\mathrm{Ca}^{2+}\right]_{\text {cyt }}$ in wild-type Arabidopsis leaves.

\section{The disturbance of calcium homeostasis in phot2 and phot1phot2 Arabidopsis mutants}

In both phototropin mutants, the effect of blue light on calcium content present in wild-type plants, was abolished. Calcium precipitates lacked multilayer structures. In the photlphot 2 mutant, precipitates rarely occurred adjacent to the chloroplast envelope and the tonoplast, where they were common in wild-type cells (Fig. 7B). Semi-quantitative analysis of the calcium content in the precipitates at the periphery of mesophyll cells confirms microscopic observations. The amount of calcium in the examined regions in both mutants was significantly higher in darkness (Fig. 6). Also the number and area of precipitates in the vacuole in darkness were higher in mutants than in wild-type plants (Fig. 7B). Both lines of evidence point to disturbed calcium homeostasis in the studied phototropin mutants and suggest that phot 2 may also regulate calcium content in darkness. Phot 2 can indeed actively function in cells even in the absence of light, as shown for the dark positioning of chloroplasts (Suetsugu et al., 2005).

In wild-type Arabidopsis mesophyll cells, blue light causes accumulation of calcium in the examined regions. This effect is negligible in the phot 2 mutant and absent in the phot1phot 2 mutant, indicating that in strong blue light calcium transport between the symplast and apoplast depends mainly on phot2. According to Harada et al. (2003), phot1 controls changes in $\left[\mathrm{Ca}^{2+}\right]_{\text {cyt }}$ in leaves only in the range of $1-50 \mu \mathrm{mol} \mathrm{m}^{-2} \mathrm{~s}^{-1}$ of blue light. This study confirms that blue light of $100 \mu \mathrm{mol} \mathrm{m}{ }^{-2} \mathrm{~s}^{-1}$ does not affect phot1-mediated calcium signatures in Arabidopsis mesophyll, as no calcium elevation in precipitates at the cell periphery was observed in the phot 2 mutant.

Changes in calcium patterns generated after red light were observed in wild-type and phot 2 plants, but not in the double mutant. The effect of red light on loosely bound calcium has been previously reported by Tretyn et al. (1992), who observed an increase in the number of KPA precipitates outside the plasma membrane and in the ER cisternae after $5 \mathrm{~min}$ red light irradiation in oat coleoptiles. This suggests that red light irradiation leads to $\mathrm{Ca}^{2+}$ removal from the cytoplasm. In the present study, a similar effect was observed in Arabidopsis, but its magnitude was substantial only in the phot 2 mutant. The mechanism by which the presence of phot 2 reduces the red light effect on $\mathrm{Ca}^{2+}$ localization remains elusive. Direct interaction between phytochromes and phototropins may be involved, as Arabidopsis phyA and phot1 were shown to interact at the plasma membrane (Jaedicke et al., 2012). Its physiological relevance seems likely when considering the effect of red light on the cytoskeletal organization observed in the phot 2 background. In wild-type Arabidopsis the organization of the cortical actin cytoskeleton is similar in the blue and red light-irradiated mesophyll cells (Krzeszowiec et al., 2007). In contrast, in the phot 2 mutant, strong red light causes a distinct shortening of actin filaments. Thus, phot 2 together with a red light photoreceptor has been suggested to control F-actin organization. Phytochrome B might be this photoreceptor, as it has been proposed to attenuate the signaling pathway leading to the chloroplast avoidance response controlled by phot2 (Luesse et al., 2010). The results presented herein suggest the involvement of calcium in arranging the actin cytoskeleton under red light in the phot 2 mutant.

Calcium homeostasis is severely disturbed in the phot 1phot 2 mutant, which is reflected in the lack of light-specific changes in calcium content at the periphery of mesophyll cells. This confirms the involvement of phototropins in controlling calcium levels in mesophyll cells after light treatment.

\section{Phototropin2-dependent chloroplast calcium patterns}

Although precipitates adjacent to the chloroplast envelope and to the tonoplast were observed in several experimental groups, they were particularly abundant after blue light in Arabidopsis wild-type cells. In the double phot 1phot 2 mutant, 
these structures were less frequently observed, thus their formation and localization seems to depend on the presence of phototropins. The lack of phot 2 causes a non-specific, lightindependent formation of precipitates inside the cell. This observation is consistent with the proposed role of internal calcium stores in the phot 2 signal transduction pathway (Harada et al., 2003). Internal $\mathrm{Ca}^{2+}$ stores are involved in the control of chloroplast movements, as was shown by inhibitor studies (Tlałka and Gabrys, 1993; Tlałka and Fricker, 1999; Aggarwal et al., 2013a). It is possible that the identified calcium precipitate patterns result from a response to blue light generated by the chloroplast. The mechanism by which the chloroplast synchronizes the direction of movement with its physiological state; that is, the efficiency of photosynthesis or the photo-oxidative damage risk, is unknown. Calcium may be a good candidate because light modulates its homeostasis inside the chloroplast. A light-dependent calcium influx into the chloroplast has been reported (for a review, see Johnson et al., 2006). On the other hand, a thylakoid protein, CAS (a $\mathrm{Ca}^{2+}$-sensing receptor) is responsible for calcium elevation in the cytoplasm (Nomura et al., 2008), showing that the chloroplast may generate calcium signatures in the cell. In Lemna, an increase in the level of calcium in cell wall areas neighboring with chloroplasts occurs during the chloroplast avoidance response chemically induced by lead (Samardakiewicz et al., 2015). In this work, almost no precipitates have been observed inside chloroplasts. This may be due to the inaccessibility of calcium to pyroantimonate precipitation in chloroplasts, where calcium is mainly bound to thylakoid membranes and stromal proteins (Stael et al., 2012).

\section{Calcium distribution in the context of chloroplast movements}

In mesophyll cells, chloroplast movements are the main phototropin-dependent responses to blue light. Several lines of evidence show that $\mathrm{Ca}^{2+}$ is involved in the signaling from phototropins to chloroplasts in different species (see Banaś et al., 2012). This study shows that calcium relocalization after blue light treatment requires the presence of phot2, as it is absent in the phot 2 mutant. This mutant also lacks full chloroplast avoidance in strong light, since phot1 alone can trigger only residual avoidance, followed by accumulation (Luesse et al., 2010; Labuz et al., 2015). Thus the observed calcium localization pattern appears to be important for eliciting the full chloroplast avoidance response.

\section{Acknowledgements}

The study of the impact of light on calcium homeostasis was supported by a grant from the Polish National Science Center [grant no. 2011/01/N/ $\mathrm{NZ3} / 00280]$. The 3D model of calcium precipitates was funded within the EU framework of FP7, Marie Curie ITN CALIPSO [GA 2013-ITN-607-607], and a grant from the Polish National Science Center [grant no. 2011/01/B/ $\mathrm{NZ3/02160].} \mathrm{JL} \mathrm{is} \mathrm{a} \mathrm{beneficiary} \mathrm{of} \mathrm{funding} \mathrm{from} \mathrm{the} \mathrm{Jagiellonian} \mathrm{University}$ within the SET project (co-financed by the European Union). The Faculty of Biochemistry, Biophysics and Biotechnology of the Jagiellonian University is a partner of the Leading National Research Center (KNOW) supported by the Ministry of Science and Higher Education, and benefits from structural funds from the European Union [grant no. POIG.02.01.00-12-064/08].
The microscopic studies were performed in the Laboratory of Electron Microscopy, Nencki Institute of Experimental Biology Warsaw, Poland and were supported by statutory funds to this Institute. The equipment used in this Laboratory: JEM1400 (JEOL Co., Japan) combined with EDS INCA Energy TEM (Oxford Instruments, UK), a tomographic holder, and a MORADA CCD camera (SiS Olympus, Germany) was financed by EU Structural Funds, project: Centre of Advanced Technology BIM Equipment purchase for the Laboratory of Biological and Medical Imaging. The authors would like to thank Rafał Bartosiewicz, Szymon Suski, and Henryk Bilski for technical assistance during microscopic studies.

\section{References}

An Q, Hückelhoven R, Kogel KH, Van Bel AJE. 2006. Multivesicular bodies participate in a cell wall-associated defense response in barley leaves attacked by the pathogenic powdery mildew fungus. Cellular Microbiology 8, 1009-1019.

Aggarwal C, Łabuz J, Gabryś H. 2013a. Phosphoinositides play differential roles in regulating phot1- and phot2-mediated chloroplast movements in Arabidopsis. PLoS One 8, e55393.

Aggarwal C, Łabuz J, Gabryś H. 2013b. Decoding the role of phosphoinositides in phototropin signaling involved in chloroplast movements. Plant Signaling and Behavior 8, e25105.

Aggarwal C, Banaś AK, Kasprowicz-Maluśki A, Borghetti C, Łabuz J, Dobrucki J, Gabryś H. 2014. Blue-light-activated phototropin2 trafficking from the cytoplasm to Golgi/post-Golgi vesicles. Journal of Experimental Botany 65, 3263-3276.

Anielska-Mazur A, Bernaś T, Gabryś H. 2009. In vivo reorganization of the actin cytoskeleton in leaves of Nicotiana tabacum $\mathbf{L}$. transformed with plastin-GFP: correlation with light-activated chloroplast responses. BMC Plant Biology 9, 1-14.

Batistič O, Kudla J. 2012. Analysis of calcium signaling pathways in plants. Biochimica et Biophysica Acta 1820, 1283-1293.

Babourina O, Newman I, Shabala S. 2002. Blue light-induced kinetics of $\mathrm{H}^{+}$and $\mathrm{Ca}^{2+}$ fluxes in etiolated wild-type and phototropin-mutant Arabidopsis seedlings. Proceedings of the National Academy of Sciences, USA 99, 2433-2438.

Banaś AK, Aggarwal C, Łabuz J, Sztatelman O, Gabryś H. 2012. Blue light signalling in chloroplast movements. Journal of Experimental Botany 63, 1559-1574.

Baum G, Long JC, Jenkins GI, Trewavas AJ. 1999. Stimulation of the blue light phototropic receptor NPH1 causes a transient increase in cytosolic $\mathrm{Ca}^{2+}$. Proceedings of the National Academy of Sciences, USA 96, 13554-13559.

Christie JM. 2007. Phototropin blue-light receptors. Annual Review of Plant Biology 58, 21-45.

Dodd AN, Kudla J, Sanders D. 2010. The language of calcium signaling. Annual Review of Plant Biology 61, 593-620.

Folta KM, Lieg EJ, Durham T, Spalding EP. 2003. Primary inhibition of hypocotyls growth and phototropism depend differently on phototropinmediated increases in cytoplasmic calcium induced by blue light. Plant Physiology 133, 1464-1470.

Harada A, Sakai T, Okada K. 2003. Phot1 and phot2 mediate blue lightinduced transient increases in cytosolic $\mathrm{Ca}^{2+}$ differently in Arabidopsis leaves. Proceedings of the National Academy of Sciences, USA 100, 8583-8588.

Harada A, Shimazaki K. 2007. Phototropins and blue light-dependent calcium signaling in higher plants. Photochemistry and Photobiology $\mathbf{8 3}$, 102-111.

Johnson CH, Shingles R, Ettinger WF. 2006. Regulation and role of calcium fluxes in the chloroplast. In Wise RR, Hoober JK, eds. The structure and function of plastids. Berlin: Springer, 403-416.

Jaedicke K, Lichtenthäler AL, Meyberg R, Zeidler M, Hughes J. 2012. A phytochrome-phototropin light signaling complex at the plasma membrane. Proceedings of the National Academy of Sciences, USA 109, 12231-12236.

Kadota A, Wada M. 1992. Photoorientation of chloroplasts in protonemal cells of the fern Adiantum as analyzed by use of a video-tracking system. Journal of Plant Research 105, 265-279.

Krzeszowiec W, Rajwa B, Dobrucki J, Gabryś H. 2007. Actin cytoskeleton in Arabidopsis thaliana under blue and red light. Biology of the Cell 99, 251-260. 
Łabuz J, Hermanowicz P, Gabryś H. 2015. The impact of temperature on blue light induced chloroplast movements in Arabidopsis thaliana. Plant Science 239, 238-249.

Luesse DR, DeBlasio SL, Hangarter RP. 2010. Integration of phot1, phot2, and PhyB signalling in light-induced chloroplast movement. Journal of Experimental Botany 61, 4387-4397.

McAinsh MR, Pittman JK. 2009. Shaping the calcium signature. New Phytologist 181, 275-294.

Miyawaki A, Griesbeck O, Heim R, Tsien RY. 1999. Dynamic and quantitative $\mathrm{Ca}^{2+}$ measurements using improved cameleons. Proceedings of the National Academy of Sciences, USA 96, 2135-2140.

Musetti R, Favali MA. 2003. Cytochemical localization of calcium and X-ray microanalysis of Catharanthus roseus L. infected with phytoplasmas. Micron 34, 387-393.

Nomura H, Komori T, Kobori M, Nakahira Y, Shiina T. 2008. Evidence for chloroplast control of external $\mathrm{Ca}^{2+}$-induced cytosolic $\mathrm{Ca}^{2+}$ transients and stomatal closure. The Plant Journal 53, 988-998.

Rossbacher R, Wagner G. 1984. X-ray microanalysis of calcium in fixed and in shock-frozen hydrated green algal cells: Mougeotia, Spirogyra and Zygnema. Nuclear Instruments and Methods in Physics Research Section B 3, 664-666.

Sakai T, Kagawa T, Kasahara M, et al. 2001. Arabidopsis nph1 and npl1: blue light receptors that mediate both phototropism and chloroplast relocation. Proceedings of the National Academy of Sciences, USA 98, 6969-6974.

Samardakiewicz S, Krzeszowiec-Jeleń W, Bednarski W, Jankowski A, Suski S, Gabryś H, Woźny A. 2015. Pb-induced avoidance-like chloroplast movements in fronds of Lemna trisulca L. PLoS One 10, e0116757.

Sato Y, Wada M, Kadota A. 2001. External $\mathrm{Ca}^{2+}$ is essential for chloroplast movement induced by mechanical stimulation but not by light stimulation. Plant Physiology 127, 497-504.

Sato Y, Wada M, Kadota A. 2003. Accumulation response of chloroplasts induced by mechanical stimulation in bryophyte cells. Planta 216, 772-777.

Schonbohm E, Schonbohm E, Meyer-Wegener J. 1990. On the signaltransduction chains of two Pfr-mediated short-term processes: increase of anchorage and movement of Mougeotia chloroplasts. Photochemistry and Photobiology 52, 203-209.
Spurr AR. 1969. A low viscosity epoxy resin embedding medium for electron microscopy. Journal of Ultrastructure Research 26, 31-43.

Suetsugu N, Kagawa T, Wada M. 2005. An auxilin-like J-domain protein, JAC1, regulates phototropin-mediated chloroplast movement in Arabidopsis. Plant Physiology 139, 151-162.

Stael S, Wurzinger B, Mair A, Mehlmer N, Vothknecht UC, Teige M. 2012. Plant organellar calcium signaling: an emerging field. Journal of Experimental Botany 63, 1525-42.

Stoelzle S, Kagawa T, Wada M, Hedrich R, Dietrich P. 2003. Blue light activates calcium-permeable channels in Arabidopsis mesophyll cells via the phototropin signaling pathway. Proceedings of the National Academy of Sciences, USA 100, 1456-1461.

Takagi S, Nagai R. 1983. Regulation of cytoplasmic streaming in Vallisneria mesophyll cells. Journal of Cell Science 62, 385-405.

Tlałka M, Fricker M. 1999. The role of calcium in blue-light-dependent chloroplast movement in Lemna trisulca L. The Plant Journal 20, 461-473.

Tlałka M, Gabryś H. 1993. Influence of calcium on blue-light-induced chloroplast movement in Lemna trisulca L. Planta 189, 491-498.

Tretyn A, Kendrick RE, Kopcewicz J. 1992. Cytochemical studies on phytochrome-mediated changes of $\mathrm{Ca}^{2+}$ localization in etiolated oat coleoptile cells. Journal of Experimental Botany 43, 439-448.

Trojan A, Gabryś H. 1996. Chloroplast distribution in Arabidopsis thaliana depends on light conditions during growth. Plant Physiology 111, 419-425.

Wagner G, Klein K. 1981. Mechanism of chloroplast movement in Mougeotia. Protoplasma 109, 169-185.

Wagner G, Rossbacher R. 1980. X-ray microanalysis and chlorotetracycline staining of calcium vesicles in the green alga Mougeotia. Planta 149, 298-305.

Wagner G, Valentin P, Dieter P, Marm D. 1984. Identification of calmodulin in the green alga Mougeotia and its possible function in chloroplast reorientational movement. Planta 152, 62-57.

Wick SM, Hepler PK. 1982. Selective localization of intracellular $\mathrm{Ca}^{2+}$ with potassium antimonate. Journal of Histochemistry and Cytochemistry 30, 1190-1204. 